\title{
Moduli spaces, non-commutative geometry and deformed differential categories
}

\author{
Ivan Verkelov \\ Research Group-Tescha, Dept. of Mathematics, Baikov Institute, Baikov, Russia
}

\section{Email address:}

verkelovi@gmail.com

\section{To cite this article:}

Ivan Verkelov. Moduli Spaces, Non-Commutative Geometry and Deformed Differential Categories. Pure and Applied Mathematics Journal. Special Issue: Integral Geometry Methods on Derived Categories in the Geometrical Langlands Program. Vol. 3, No. 6-2, 2014, pp. 12-19. doi: $10.11648 /$ j.pamj.s.2014030602.13

\begin{abstract}
The following research plays a central role in deformation theory. If $\mathrm{x}$, is a moduli space over a field $k$, of characteristic zero, then a formal neighborhood of any point $x \in \mathrm{x}$, is controlled by a differential graded Lie algebra. Then using the derived categories language we give an analogous of the before sentence in the setting of non-commutative geometry, considering some aspects $\mathrm{E}_{\infty}$ - rings and derived moduli problems related with these rings. After is obtained a scheme to spectrum; by functor Spec and their $\infty$ - category functor inside of the space Fun $_{\text {hoat }_{\infty}}$ to these $\mathrm{E}_{\infty}$ - rings and their derived moduli in field theory.
\end{abstract}

Keywords: Deformed Category, $\mathrm{E}_{\infty}$ — Rings, Formal Moduli Problem, Koszul Duality, Non-Commutative Geometry

\section{Introduction}

The idea begin with the research developed of a unpublished work of Deligne, Drinfeld, Feigin, and has powerfully influenced subsequence contributions, for example, the Kontsevich-Soibelman work, or the Manetti work, being others.

However, we will adopt Grothendieck's "functor of points" philosophy [1], giving to the moduli space $X$, as equivalent to specifying the functor $R \rightarrow X(R)=\operatorname{Hom}(\operatorname{Spec} R, X)$. We will consider several variations on this theme:

a) Allowing $R$, to range over the categoru ring of commutative rings, we obtain the notion of a classical moduli problem (Definition 2. 3). We will discuss this notion and give several examples in $\S 1$.

b) To understand the deformation theory of a moduli space $X$, it is often useful to extend the definition of the functor $R \rightarrow X(R)$, to a more general class of rings. The algebraic topology provides such a generalizations via the theory of $E_{\infty}$ - rings spectra.

c) Let $k$, be a field. To study the local structure of a moduli space $X$, near a point $x \in X(k)$, it is useful to restrict our attention to the values $X(R)$, where $R$, is a ring which is, in some sense, very similar to $k$ (for example, local Artin algebras having residue field $k$ ). In this part will make this precise by introducting the notion of a formal moduli problem (Definition 5. 6).

d) Anoter way of enlarging the category of commutative rings is by weakening the requirement of commutativity. In the setting of ring spectra there are several flavors of commutativity available, giving by the theory of $E_{n}$ rings for $0 \leq n<\infty$. We will define the notion of a formal $E_{n}$-moduli problem.

\section{Moduli Problems for Commutative Rings}

Let $\mathrm{R}$, denote the category of commutative rings and $\mathrm{S}$, the category of sets. Doing extensive the use of Grothendieck's "functor of points" philosophy: that is, we will identify a geometric object $X$ (such as a scheme) with the functor $\mathrm{R} \rightarrow \mathrm{S}$, represented by $X$, given by the formula $R \rightarrow \operatorname{Hom}(\operatorname{Spec} R, X)$.

We consider the following examples.

Example 2. 1. Let $X: \mathrm{R} \rightarrow \mathrm{S}$, be the functor which assigns to each commutative ring $R$, the set $R^{\times}$. Of invertible elements of $R$. For any commutative ring $R$, we have a canonical bijection $X(R)=R^{\times} \cong \operatorname{Hom}_{\mathrm{R}}\left(\mathbf{Z}\left[t^{ \pm 1}\right], R\right)$. In other 
words, we can identify $X$, with the functor represented by the commutative ring $\mathbf{Z}\left[t^{ \pm 1}\right]$.

Example 2. 2. We fix an integer $n \geq 0$. We define a functor $X: \mathrm{R} \rightarrow \mathrm{S}$, by letting $F(R)$, denote the set of all submodules $M \subseteq R^{n+1}$, such that the quotient $R^{n+1} / M$, is a projective $R$-module of rank $n$ (from which it follows that $M$, is a projective $R$-module of rank 1). The functor $X$, is not representable by a commutative ring. However, it is representable in the large category Sch, of schemes. That is, for any commutative ring $R$, we have a canonical bijection

$$
X(R) \cong \operatorname{Hom}_{\mathrm{Sch}}\left(\operatorname{Spec} R, \mathrm{P}^{n}\right)
$$

where $\mathrm{P}^{n} \cong \operatorname{Proj} \mathbf{Z}\left[x_{0}, \ldots, x_{n}\right]$, denotes projective space of dimension $n$.

For our proposes, the action of a functor $X: \mathrm{R} \rightarrow \mathrm{S}$, is too restrictive. We often want to study moduli problems $X$, which assign to a commutative ring $R$, some class of geometric objects which depend on $R$. The trouble is that this collection of geometrical objects is naturally organized into a category, rather than a set. This motivates the following definition:

Definition 2. 1. Let Gpd, denote the collection of grupoids, that is to say categories in which every morphism is an isomorphism. We regard Gpd, as a 2-category, that is to say, morphisms are given by functors between grupoids and 2 - morphisms are given by natural transformations (which are automatically invertible). A classical moduli problem is a functor $X: \mathrm{R} \rightarrow \mathrm{Gpd}$.

Every set $S$, can be regarded as a grupoid by setting

$$
\operatorname{Hom}_{S}(x, y)=\left\{\begin{array}{c}
\left(\mathrm{id}_{x}\right), \text { if } x=y \\
\varnothing, \text { if } x \neq y
\end{array}\right.
$$

This construction allows us to identify the category $S$, with a full subcategory of the 2 - category Gpd. In particular, every functor $X: \mathrm{R} \rightarrow \mathrm{S}$, can be identified with a classical moduli problem in the sense of the definition 2. 1 .

Example 2. 3. For every commutative ring $R$, let $X(R)$, be the category of elliptic curves $E \rightarrow \operatorname{Spec} R$ (morphisms in the category $X(R)$, are given by isomorphisms of elliptic curves). Then $F$, determines a functor $\mathrm{R} \rightarrow \mathrm{Gpd}$, and can therefore be regarded as a moduli problem in the sense of the definition 2. 1. This moduli problem cannot be represented by a commutative ring or even by a scheme, that is to say, for any scheme $Y$, the space $\operatorname{Hom}_{\mathrm{Sch}}(\operatorname{Spec} R, Y)$, is a set. In particular if we remember the space $\operatorname{Hom}_{\mathrm{Sch}}(\operatorname{Spec} R, Y)$, as a grupoid, every object has a trivial automorphism group. In contrast, every object of $X(R)$, has a non-trivial automorphism group, that is to say, every elliptic curve admits a nontrivial automorphism, given by multiplication by -1 .

Nevertheless, the moduli problem, is representable if we work not in the category of schemes but in the larger 2category $\mathrm{St}_{\mathrm{DM}}$, of Deligne-Mumford stacks. More precisely, there exists a Deligne-Mumford stack $\mathrm{M}_{\text {Ell }}$ (the moduli stack of elliptic curves) for which there is a canonical equivalence of categories

$$
X(R) \cong \operatorname{Hom}_{\mathrm{St}_{\mathrm{DM}}}\left(\operatorname{Spec} R, \mathrm{M}_{\mathrm{Ell}}\right)
$$

for every commutative ring $R$.

Example 2. 4. We fix an integer $n \geq 0$. For every commutative ring $R$, let $X(R)$, denote the category whose objects are projective $R$-modules of rank $n$, and whose morphisms are given by isomorphisms of $R$-modules. Then $X$, can be regarded as a moduli problem $\mathrm{R} \rightarrow \mathrm{Gpd}$. This moduli problem is not representable in the 2 - category $\mathrm{St}_{\mathrm{DM}}$, of Deligne-Mumford stacks [2], because projective $R-$ modules admit continuous families of automorphisms. However, $F$, is representable in the larger 2-category $\mathrm{St}_{\mathrm{Art}}$, of Artin stacks. Namely, there is an Artin stack $\operatorname{BGL}(n) \in \mathrm{St}_{\mathrm{Art}}$, for which there is a canonical bijection

$$
X(R) \cong \operatorname{Hom}_{\mathrm{St}_{\mathrm{Art}}}(\operatorname{Spec} R, \operatorname{BGL}(n)),
$$

for every commutative ring $R$.

\section{Higher Categories Theories}

In the before section 2, we discussed the notion of a moduli problem in classical algebraic geometry. Even very simple moduli problem involve the classification of geometric objects which admit nontrivial automorphisms, and should therefore be treated as categories rather than as sets (Examples 2. 3, and 2. 4). Consequently, moduli problems themselves (and the geometric objects which represent them) are organized not into a category, but into a 2-category. Our discussion in this paper will take us much further into the realm of higher categories.

Definition 3. 1. Let $n \geq 0$, be a nonnegative integer. The notion of an $n$-category is defined by induction on $n$. If $n=0$, an $n$ - category is simply a set. If $n>0$, an $n-$ category C, consists of the following:

(1) A collection of objects $X, Y, Z, \ldots$

(2) For every pair of objects $X, Y \in \mathrm{C}$, an $(n-1)-$ category $\operatorname{Hom}_{\mathrm{C}}(X, Y)$.

(3) Composition laws given by

$$
\phi_{\mathrm{X}, \mathrm{Y}, \mathrm{Z}}: \operatorname{Hom}_{\mathrm{c}}(X, Y) \times \operatorname{Hom}_{\mathrm{c}}(Y, Z) \rightarrow \operatorname{Hom}_{\mathrm{c}}(X, Z),
$$

which are require to be unital and associative.

If $\eta$, is an object of the $(n-1)$ - category $\operatorname{Hom}_{\mathrm{C}}(X, Y)$, for some pair of objects $X, Y \in \mathrm{C}$, then we will say that $\eta$, is a 1 -morphism of $\mathrm{C}$. More generally, a $k$-morphism in $\mathrm{C}$, is $\mathrm{a}(k-1)$-morphism in some $(n-1)$ - category $\operatorname{Hom}_{\mathrm{C}}(X, Y)$.

Example 3. 1. Every topological space $X$, determines an $n$ - category $\pi_{\leq n} X$, the fundamental grupoid of $X$. If $n=0$, we let $\pi_{\leq n} X=\pi_{0} X$, be the set of path components of 
$X$. For $n>0$, we let $\pi_{\leq n} X$, be the $n$-category whose objects are points of $X$, where $\operatorname{Hom}_{\pi_{\leq n}}(x, y)$, is the fundamental $(n-1)$-grupoid $\pi_{\leq n-1} P_{x, y}(X)$, where

$$
P_{x, y}(X)=\{p:[0,1] \rightarrow X \mid p(0)=x, p(1)=y\}
$$

is the space of paths from $x$, to $y$, in $X$. Composition in $\pi_{\leq n} X$, is given by concatenation of paths. If $n=1$, this definition recovers the usual fundamental grupoid of $X$.

The definition 3. 1, is informal because we did not specify precisely what sort of associative law the composition in $\mathrm{C}$, is required to satisfy. If $n=1$, there is no real ambiguity and the definition 3. 1, recovers the usual definition of a category. When $n=2$, the situation is more subtly, that is to say, the associative law should posit the commutativity of a diagram having the form:

$$
\begin{aligned}
\operatorname{Hom}_{\mathrm{c}}(W, X) \times \operatorname{Hom}_{\mathrm{c}}(X, Y) \times \operatorname{Hom}_{\mathrm{c}}(Y, Z) \stackrel{\phi_{W, X Y}}{\longrightarrow} & \operatorname{Hom}_{\mathrm{c}}(W, X) \times \operatorname{Hom}_{\mathrm{c}}(Y, Z) \\
& \downarrow \phi_{X, Y, Z} \\
& \downarrow \phi_{W, Y, Z} \\
& \operatorname{Hom}_{\mathrm{c}}(W, X) \times \operatorname{Hom}_{\mathrm{c}}(X, Z) \stackrel{\phi_{w, X Z}}{\longrightarrow} \operatorname{Hom}_{\mathrm{c}}(W, Z),
\end{aligned}
$$

Since this is a diagram of categories and functors, rather than sets and functions, we are faced with a question, do we require this diagram to commute "on the nose" or only up to isomorphism? In the former case, we obtain the definition of a strict 2 -category. This generalizes in a straightforward way, that is to say, we can require strict associativity in the definition 3. 1, to obtain a notion of strict $n$-category for every $n$. However, this notion turns out to be of limited use. For example, the fundamental $n$-groupoid of a topological space $\pi_{\leq n} X$, usually cannot be realized as a strict $n$-category when $n>2$. In the example 3.1 , it is necessary to interpret Definition 3. 1., differently. In place of equality, we require the existence of isomorphisms

$$
\begin{aligned}
& \gamma_{W, X, Y, Z}: \phi_{W, X, Z} \circ\left(\mathrm{id}_{\mathrm{HomC}(W, X)} \times \phi_{\mathrm{X}, \mathrm{Y}, \mathrm{Z}}\right) \cong \\
& \cong \phi_{W, Y, Z} \circ\left(\phi_{W, X, Y} \times \mathrm{id}_{\mathrm{HomC}(\mathrm{Y}, Z)}\right),
\end{aligned}
$$

These isomorphisms are themselves part of the structure C, and are required to satisfy certain coherence conditions. When $n>2$, these coherence conditions are themselves only required to hold up to isomorphism, being these isomorphism specified and required to satisfy further coherences, and so forth. As $n$, grows, it becomes prohibitively difficult so specify these coherences explicitly.

One thesis to respect was enounced establishing that:

"The construction $X \rightarrow \pi_{\leq n} X$, establishes a bijective correspondence between $n$-types (up to weak homotopy equivalence) and $n$ - grupoids (up to equivalence)",

We enounce the following definition.

Definition 3. 2. A topological category is a category c, for which each of the sets $\operatorname{Hom}_{\mathrm{C}}(X, Y)$, is equipped with a topology, and each of the composites maps (3.1) is continuous. If $\mathrm{C}$, and $\mathrm{D}$, are topological categories, we will say that a functor $F: \mathrm{C} \rightarrow \mathrm{D}$, is continuous if, for every pair of objects
$X, Y \in \mathrm{C}$, the map

$$
\operatorname{Hom}_{\mathrm{C}}(X, Y) \rightarrow \operatorname{Hom}_{\mathrm{C}}(F X, F Y)
$$

is continuous. The collection of "small" topological categories and continuous functors forms a category, which we will denote by Cat $_{t}$.

Example 3. 2. Let C, and D, be $\infty$-categories. Then there exists another $\infty$ - categories Fun(CD), with the following universal property of that for every $\infty$ - category there is a canonical bijection given as

$$
\left.\operatorname{Hom}_{\mathrm{hGat}_{\infty}}(\mathrm{C}, \mathrm{Fun}(\mathrm{C}, \mathrm{D})) \cong \operatorname{Hom}_{\mathrm{Cat}_{\infty}}(\mathrm{C} \times \mathrm{C}, \mathrm{D})\right),
$$

We will refer to objects of Fun(C,D), simply as functors from $C$, to $D$.

\section{Rings and their Spectrums}

Let $\mathrm{C}$, be the category of all topological spaces and let, be the collection of weak homotopy equivalences. We will refer to $\left.q W^{-1}\right]$, as the $\infty$-category of spaces and denote it by $\mathrm{S}$. We describe the object of $S$, as the $\mathrm{CW}$ complexes [3], and whose property between $\mathrm{CW}$ complexes is:

(a) The objects of S, are CW complexes.

(b) For every pair of $\mathrm{CW}$ complexes $X$, and $Y$, we let $\operatorname{Hom}_{\mathrm{S}}(X, Y)$, denote the space of continuous maps from

$X$, to $Y$, (endowed with the compact-open topology).

The role of $S$, in the theory of $\infty-$ categories is analogous to that of the ordinary category of sets in classical category theory. For example, for any $\infty-$ category $C$, one can define a Yoneda embedding [4]

$$
j: \mathrm{C} \rightarrow \operatorname{Fun}\left(\mathrm{C}^{\text {top }}, \mathrm{S}\right)
$$

given explicitly by

$$
j(C) D=H o m_{\mathrm{C}}(D, C) \in \mathrm{S},
$$

\footnotetext{
${ }^{1}$ The construction of this category $\mathrm{Cat}_{t}$, can be established as: let $\mathrm{C}$, be a topological category. We can associate to $\mathrm{C}$, an ordinary category $\mathrm{hC}$, as folløws The objects of $\mathrm{C}$, are the objects of $\mathrm{hC}_{\mathrm{C}}$.

- For every pair of objects $X, Y \in \mathrm{C}$, we let $\operatorname{Hom}_{\mathrm{hC}}(X, Y)=\pi_{0} \operatorname{Hom}_{\mathrm{C}}(X, Y)$,

that is, maps from $X$, to $Y$, are $\mathrm{hC}$, homotopy classes of maps from $X$, to $Y$, are $\mathrm{C}$

We say that a morphism $f$, in $\mathrm{C}$, is an equivalence if the image of $f$, in hC, is an isomorphism.

Definition. Let $F: \mathrm{C} \rightarrow \mathrm{D}$, be a continuous functor between topological categories. We will say that $F$, is a weak equivalence if the following conditions are satisfied:

a) The functor $F$, induces an equivalence of ordinary categories

b) $\mathbb{T} \in \mathbb{d r}$ pair of objects $X, Y \in \mathrm{C}$, the induced map

$$
\operatorname{Hom}_{\mathrm{C}}(X, Y) \rightarrow \operatorname{Hom}_{\mathrm{D}}(F X, F Y),
$$

is a weak homotopy equivalence.

Let $\mathrm{hCat}_{\infty}$, be the category obtained from $\mathrm{Cat}_{t}$, by formally inverting the collection of weak equivalences. A $(\infty, 1)$ - category is an object of $\mathrm{hCat}_{\infty}$. We will refer to $\mathrm{hCat}_{\infty}$, as the homotopy category of $(\infty, 1)$ - categories.
} 
In this research we are interested in the $\infty$ - category analogous more algebraic structures like commutative rings (see the Table 1).

Table 1. Analogies

\begin{tabular}{ll}
\hline Classical Structure & $\infty-$ categorical Analogue \\
\hline Set & Topological Space \\
Category & $\infty-$ category \\
Abelian Group & Spectrum \\
Commutative Ring & $E_{\infty}-$ Ring \\
Ring of Integers Z & Sphere spectrum $S$ \\
\hline
\end{tabular}

Then a functor (4. 1) to these structures is given by the functor Spec .

To establish their application in the homotopy frame we need define before the notion of stable homotopy theory.

Def. 4. 1. A spectrum is a sequence of pointed spaces $X_{1}, X_{2}, \ldots \in \mathrm{S}_{*}$, equipped with weak homotopy equivalences $X_{n} \cong \Omega X_{n+1}$; here $\Omega_{*}: \mathrm{S}_{*} \rightarrow \mathrm{S}_{*}$, denotes the based loop space functor

$$
X \mapsto\{p:[0,1] \rightarrow X \mid p(0)=p(1)=*\}
$$

To any spectrum $X$, we can associate Abelian groups $\pi_{k} X$, for every integer $k$, defined by $\pi_{k} X$, for $n \gg 0$.

We say that $X$, is connective if $\pi_{k} X \cong 0$, for $n<0$.

The collection of spectra is itself organized into an $\infty-$ category which we will denote by $\mathrm{Sp}$. If

$$
X=\left\{X_{n}, \alpha_{n} \mid X_{n} \cong \Omega X_{n+1}\right\}_{n \geq 0}
$$

Is a spectrum, then we will refer $X_{0}$, as the $0 t h$ - space of $X$. The construction determines a forgetful functor $\mathrm{Sp} \rightarrow \mathrm{S}_{*}$, which we will denote by $\Omega^{\infty}$.

We will say a spectrum $X$, is discrete if the homotopy groups $\pi_{i} X$, vanish for $i \neq 0$. The construction $X \rightarrow \pi_{0} X$, determines an equivalence from the $\infty$ - category of discrete spectra to the ordinary category of Abelian groups. In other words, we can regard the $\infty$ - category $\mathrm{Sp}$, as an enlargement of the ordinary category of Abelian groups, just as the $\infty$ - category $s$, is an enlargement of the ordinary category of sets.

The category $\mathrm{Ab}$, of Abelian groups is an example of a symmetric monoidal category, that is to say, there is a tensor product operation:

$$
\otimes: \mathrm{Ab} \times \mathrm{Ab} \rightarrow \mathrm{Ab}
$$

which is commutative and associative up to isomorphism. This operation has a counterpart in the setting of spectra, that is to say, the $\infty$-category $\mathrm{Sp}$, admits a symmetric monoidal structure:

$$
\wedge: \mathrm{Sp} \times \mathrm{Sp} \rightarrow \mathrm{Sp},
$$

This operation is called the smash product, and is compatible with the usual tensor product of Abelian groups in the following sense: if $X$, and $Y$, are connective spectra, then there is a canonical isomorphism of Abelian groups

$$
\pi_{0}(X \wedge Y) \cong \pi_{0} X \otimes \pi_{0} Y,
$$

The unit object for the smash product $\wedge$, is called the sphere spectrum and denoted for $S$.

The symmetric monoidal structure on the $\infty$ - category $\mathrm{Sp}$, allows us to define an $\infty$ - category $\mathrm{CAlg}(\mathrm{Sp})$, of commutative algebra objects of $\mathrm{Sp}$. An object of $\mathrm{CAlg}(\mathrm{Sp})$, is a spectrum $R$, equipped with the multiplication $R \wedge R \rightarrow R$, which is unital, associative and commutative up to coherent homotopy. We will refer to the objects of $\mathrm{CAlg}(\mathrm{Sp})$,

as $E_{\infty}$ - rings and to $\mathrm{CAlg}(\mathrm{Sp})$, as the $\infty$ - category of $E_{\infty}$ - rings. The sphere spectrum $S$, can be regarded as an $E_{\infty}$ - ring in an essentially unique way, and is an initial object of the $\infty$-category $\mathrm{CAlg}(\mathrm{Sp})$.

For any $E_{\infty}-\operatorname{ring} R$, the product on $R$, determines a multiplication on the direct sum $\pi_{*} R=\bigoplus_{n} \pi_{n} R$. This multiplication is unital, associative, and commutative in the graded sense (that is to say, for $x \in \pi_{i} R$, and $y \in \pi_{j} R$, we have $\left.x y=(-1)^{i j} y x \in \pi_{i+j}(R)\right)$. In particular $\pi_{0} R$, is a commutative ring and each $\pi_{i} R$, has the structure of a module over $\pi_{0} R$. The construction $R \rightarrow \pi_{0} R$, determines an equivalence between the $\infty$-category of discrete $E_{\infty}$ - rings and the ordinary category of commutative rings. Consequently, we can view $\mathrm{CAlg}(\mathrm{Sp})$, as an enlargement of the ordinary category of commutative rings.

To every $E_{\infty}$ - ring $R$, we can associate an $\infty$ - category $\operatorname{Mod}_{R}(\mathrm{Sp})$, of $R$-module spectra, that is to say, modules over $R$, in the $\infty$-category of spectra. If , and $\mathrm{N}$, are $R-$ module spectra, we will denote the space $\operatorname{Hom}_{\operatorname{Mod}_{R}(\mathrm{Sp})}(M, N)$.

Motivated for the analogies given in the table 1, we can give the following definition:

Def. 4. 2. A derived moduli problem is a functor $X$, from the $\infty$ - category $\mathrm{CAlg}(\mathrm{Sp})$, of $E_{\infty}$ - rings to the $\infty-$ category $\mathrm{S}$, of spaces.

\section{Three Examples to Field Theory}

We can consider the following two useful examples in field theory, and that can be used in the field description of the algebraic behavior of the elements of the moduli space of the Higgs fields.

Example 5. 1. Let $\mathrm{X}_{0}$, be scheme (or, more generally, an algebraic stack), and let $X_{0}$, be the classical moduli problem given by the formula $F_{0}(R)=\operatorname{Hom}\left(\operatorname{Spec} R, \mathrm{X}_{0}\right)$. Let $Y$, be a smooth algebraic variety over the complex numbers, and let $\mathrm{M}_{g}(Y)$, denote the Kontsevich moduli stack of curves of genus $g$, equipped with a stable map to $Y$ (see, for example 
[2]). Then $\mathrm{M}_{g}(Y)$, represents a functor $X_{0}: \operatorname{Ring} \rightarrow \mathrm{Gpd}$, which admits a natural enhancement $X: \mathrm{CA} \lg (\mathrm{Sp}) \rightarrow \mathrm{S}$. This enhancement contains a great deal of useful information about the original moduli stack $\mathrm{M}_{g}(Y)$, since for example, it determines the virtual fundamental class of $\mathrm{M}_{g}(Y)$, which play an important role in the Gromov-Witten theory.

Example 5. 2. Let $X_{0}$ : Ring $\rightarrow$ Gpd, be the classical moduli problem of example 2. 3, which assigns to each commutative ring $R$, the grupoid $\operatorname{Hom}\left(\operatorname{Spec} R, \mathrm{M}_{1,1}\right)$, of elliptic curves over $R$. It is possible to make sense of the notion of an elliptic curve over $R$, when $R$, is an arbitrary $E_{\infty}-$ ring, and thereby obtain an enhancement $X: \mathrm{CA} \lg (\mathrm{Sp}) \rightarrow \mathrm{S}$, of $M_{\text {Ell }}$. One can use the enhancement to give a moduli-theoretic reformulation of the Goerss-Hopkins-Miller theory of topological modular forms [5].

Example 5. 3. The framework of derived moduli problems (or more precisely, their formal analogues), see the following definition:

Def. 5. 1. Let $k$, be a field. A formal moduli problem over $k$, is a functor $X: \mathrm{CAlg}_{\mathrm{sm}} \rightarrow \mathrm{S},{ }^{2}$ with the following properties:

a) The space $X(k)$, is contractible.

b) Suppose that $\phi: A \rightarrow B$, and $\phi^{\prime}: A^{\prime} \rightarrow B$, are maps between small $\infty-$ algebras over $k$, which induce surjections $\pi_{0} A \rightarrow \pi_{0} B, \pi_{0} A^{\prime} \rightarrow \pi_{0} B$. Then the canonical map

$$
X\left(A \times_{B} A^{\prime}\right) \rightarrow X(A) \times_{X(B)} X\left(A^{\prime}\right)
$$

is a homotopy equivalence.

This provides a good setting for the study of deformation theory.

\section{Schemes to Derived Moduli Problems}

We begin with some elements of the non-commutative geometry.

Def, 6. 1. Let $k$, be a field and let $n \geq 0$, be an integer. A formal $E_{n}-$ moduli problem over $k$, is a functor

$$
X: \operatorname{Alg}_{\mathrm{sm}}^{(n)} \rightarrow \mathrm{S}
$$

${ }^{2}$ We let $\mathrm{CAlg}_{k}$, denote the $\infty$-category whose objects are $E_{\infty}$ - rings $A$, equipped with a map $k \rightarrow A$, where morphisms are given by commutative triangles:

$$
\begin{gathered}
\lambda^{k} \mu \\
A A^{\prime}
\end{gathered}
$$

We will refer to the objects of $\mathrm{CAlg}_{k}$, as $E_{\infty}$-algebras over $k$.

We let $\operatorname{Mod}_{\mathrm{sm}}$, denote the full subcategory of $\operatorname{Mod}_{k}$, spanned by the small $k-$ module spectra, and $\mathrm{CAlg}_{\mathrm{sm}}$, denote the full subcategory of $\mathrm{CAlg}_{k}$, spanned by the small $E_{\infty}$-algebras over $k$. with the following properties:

i) The space $X(k)$, is contractible.

ii) Suppose we are given a pullback diagram of small $E_{n}-$ algebras

$$
\begin{aligned}
& A^{\prime} \rightarrow A \\
& \downarrow \quad \downarrow, \\
& B^{\prime} \rightarrow B
\end{aligned}
$$

such that the maps $\pi_{0} A \rightarrow \pi_{0} B$, and $\pi_{0} B^{\prime} \rightarrow \pi_{0} B$, are surjective. Then the diagram

$$
\begin{aligned}
& X\left(A^{\prime}\right) \rightarrow X(A) \\
& \downarrow \quad \downarrow \quad \\
& X\left(B^{\prime}\right) \rightarrow X(B)
\end{aligned}
$$

is a pullback diagram in $\mathrm{s}$.

Let $V_{0}$, be a finite dimensional vector space over $k$, and let

$$
X_{x}: \mathrm{CAlg}_{\mathrm{sm}} \rightarrow \mathrm{S}
$$

be the formal moduli problem ${ }^{3}$, so that $X_{x}$, assigns to every small $E_{\infty}$-algebra $A$, ring over $k$, the $\infty$-grupoid of pairs $(V, \alpha)$, where $V$, is an $A$-module and $\alpha: k \wedge_{A} V \cong V_{0}$, is an equivalence. The definition of $X_{x}$, does not make any use of the commutativity of $A$. Consequently, $X_{x}$, extends naturally to a functor $\widetilde{X}_{x}: \operatorname{Alg}_{\mathrm{sm}}^{(1)} \rightarrow \mathrm{S}$. By definition, shifted tangent complex of $\widetilde{X}_{x}[-1]$, is given by the Lie algebra

\footnotetext{
${ }^{3}$ Let $\mathrm{CAlg}(\mathrm{Sp}) \rightarrow \mathrm{S}$, be the formal moduli problem of Artin stack $B G L(n)$, example 2.3, which assigns to every $E_{\infty}$-ring $A$, the $\infty$ - grupoid $F(A)$, of prospective $A$-modules of rank $n$. Giving a point $x=(k, \eta)$, of $X$, is equivalent to giving a field $k$ together with a vector space $V_{0}$, of dimension $n$, over $k$. In this case the functor $X_{x}: \mathrm{CAlg}_{\mathrm{sm}} \rightarrow \mathrm{S}$, can be described as follows: to every small $k$-algebra $A$, the functor $X_{x}$, assigns the $\infty$ - category of pairs $(V, \alpha)$, where $V$, is a projective $A$ - module of rank $n$, and $\alpha: k \wedge_{A} V \rightarrow V_{0}$, is an isomorphism of $k-$ vector spaces. We can denote their tangent complex as $T_{X, x}$.

We see that $T_{X, x}(0)=X_{x}\left(k[\in] /\left(\in^{2}\right)\right)$, can be identified with a classifying space for the grupoid of projective $k[\in] /\left(\epsilon^{2}\right)-$ modules $V$, which deform $V_{0}$. This grupoid has only one object up to isomorphism given by the tensor product $k[\in] /\left(\in^{2}\right) \otimes_{k} V_{0}$. It follows that $T_{X, x}(0)$, can be identified with the classifying space $B G$, for the group $G$, of automorphisms of $k[\in] /\left(\epsilon^{2}\right) \otimes_{k} V_{0}$, which reduce to the identity moduli $\in$. Such an automorphism can be written as $1+\in M$, where $M \in \operatorname{End}\left(V_{0}\right)$. Consequently $T_{X, x}(0)$, is a homotopy equivalent to the classifying space for the $k$-vector space $\operatorname{End}\left(V_{0}\right)$, regarded as a group under addition.

Then we obtain an equivalence of $k$-module spectra $T_{X, x} \cong \operatorname{End}_{k}\left(V_{0}\right)[1]$. The shifted tangent complex

$T_{X, x}[-1] \cong \operatorname{End}_{k}\left(V_{0}\right)$,
}

Has the structure of a Lie algebra over $k$ (and therefore of a differential graded Lie algebra over $k$, with trivial grading and differential), given by the usual commutator bracket of endomorphisms. 
$T_{X_{x}}[-1] \cong \operatorname{End}\left(V_{0}\right)$. If $k$, is of characteristic zero, then the formal moduli problem $X_{x}$, can be canonically reconstructed from the vector space $\operatorname{End}\left(V_{0}\right)$, together with their Lie algebra structure. For example, being $k$, a field of the characteristic zero, and Moduli, given through the full sub-category of Fun $\left(\mathrm{CAlg}_{\mathrm{sm}}, \mathrm{S}\right)$, generated by the formal moduli problems over $k$, we can establish that there is an equivalence of $\infty-$ categories given by

$$
\Phi: \text { Moduli } \rightarrow \mathrm{Lie}_{k}^{\mathrm{dg}},
$$

where $\operatorname{Lie}_{k}^{\mathrm{dg}}$, denotes the $\infty$ - category of differential graded Lie algebras over $k$.

However, the formal $E_{1}$, moduli problem $X_{x}$, is additional data, since we can evaluate $X_{x}$, on algebras which are not necessarily commutative. Then, it is natural to expect the existence of $X_{x}$, to be reflected in some additional structure on the Lie algebra $\operatorname{End}\left(V_{0}\right)$. But with a major careful we can to see that $\operatorname{End}\left(V_{0}\right)$, is not merely a Lie algebra, since, there is an associative product (given by composition) whose commutator gives the Lie bracket on $\operatorname{End}\left(V_{0}\right)$. Then is had the result:

Theorem 6.1. Let $k$, be a field, let $n \geq 0$, and let Moduli $_{n}$, be the full sub-category of $\operatorname{Fun}\left(\operatorname{Alg}_{\mathrm{sm}}^{(n)}, \mathrm{S}\right)$, spanned by the formal $E_{n}$, moduli problem. Then there exists an equivalence of $\infty$ - categories

$$
\Phi: \operatorname{Moduli}_{n} \rightarrow \operatorname{Alg}_{\text {aug }}^{(n)}
$$

Moreover, if $U: \mathrm{Alg}_{\text {aug }}^{(n)} \rightarrow \operatorname{Mod}_{k}$, denotes the forgetful functor $A \mapsto \mathrm{m}_{A}$, which assigns to each augmented $E_{n}-$ algebra their augmentation ideal, then the composition $U \circ \Phi$, can be identified with the functor $X \mapsto T_{X}[-n]$.

Proof. [6-8, 9-13].

\section{Results}

Let $A, B \in \operatorname{Alg}_{\text {aug }}^{(n)}$. Let $X \in$ Moduli $_{n}$, the formal $E_{n}-$ moduli problems on $k$. Let $\Phi_{\mathrm{R}} \mathrm{F}, \mathrm{G} \Psi_{\mathrm{R}}, \forall \mathrm{R}$ the functors defined and determined by the theorem 6. $1, \forall \mathrm{R}$, a $k-$ module that is an $A$-module. Then the isomorphism that represent these functors, are translated in the equivalences of $\infty$ - categories:

$$
\operatorname{Moduli}_{n} \underset{\mathrm{G} \Psi_{\mathrm{R}}}{\stackrel{\Phi_{\mathrm{R}} \mathrm{F}}{\Delta}} \operatorname{Alg}_{\text {aug }}^{(n)}
$$

which is true by the theorem 6. 1 .

We consider the following definition to establish the existence of the adjunct left and right functors $F, G$, that appear by integral transforms that are involved in the $k-$ modules level [14].

Def. 7. 1. Let $X \in$ Moduli $_{n}$, be a formal $E_{n}$ - moduli problems over $k$, and let $A$, be an augmented $E_{n}$-algebra over $A$. We will say that a natural transformation

$$
\alpha: X \rightarrow \Psi(A)
$$

reflects $X$, if, for every augmented $E_{n}$-algebra $B$, over $k$, composition with $\alpha$, induces a homotopy equivalence:

$$
\operatorname{Hom}_{\mathrm{Alg}_{\text {aug }}^{(n)}}(A, B) \rightarrow \operatorname{Hom}_{\text {Moduli } n_{n}}(X, \Psi(B)),(
$$

We let Moduli $_{n}$, denote the full sub-category of Moduli ${ }_{n}$, spanned by these formal $E_{n}$ - moduli problems $X$, for which there exists a map (7.2) which reflects F. In this case, the map (7. 2) is well-defined up to canonical equivalence, considering in particular that we can regard the construction $X \mapsto A$, as defining a functor

$$
\Phi: \text { Moduli }_{n}^{\mathrm{o}} \rightarrow \mathrm{Alg}_{\text {aug }}^{(n)},
$$

The functor $\Phi$, is left adjunt to $\Psi$, in the sense that for every $X \in \mathrm{Moduli}_{n}^{\circ}$, and every $B \in \mathrm{Alg}_{\text {aug }}^{(n)}$, we have a canonical homotopy equivalence

$$
\operatorname{Hom}_{\operatorname{Moduli}_{n}}(X, \Psi(B)) \rightarrow \operatorname{Hom}_{\operatorname{Alg}_{\text {aug }}^{(n)}}(\Phi(X), B),(7.5
$$

Indeed, since the functor $\Psi: \mathrm{Alg}_{\text {aug }}^{(n)} \rightarrow$ Moduli $_{n}$, preserves small limits, one can deduce the existence of a left adjoint to $\Psi$, using the adjoint functor theorem. In other words, it follows formally that Moduli $_{n}^{\circ}=$ Moduli $_{n}$. However, we will establish this equality by a more direct argument, which will help us to compute with the functor $\Phi$.

Also we consider the following property due to Koszul self-duality of the little $n$-cubes operad $[11,15]$ :

$$
\mathrm{A} \lg _{\mathrm{aug}}^{(n)} \stackrel{\Phi^{-1}}{\cong} \operatorname{Moduli}_{n} \subseteq \operatorname{Fun}\left(\operatorname{Alg}_{\mathrm{sm}}^{(n)}, \mathrm{S}\right)
$$

Theorem (I. Verkelov, F. Bulnes) 7. 1. Considering the functors $\Phi, \Psi$, with the before properties (7. 1), (7. 3), (7. 5) and (7.6), we have the following scheme

$$
\operatorname{Hom}_{\text {Moduli } n}(X, \operatorname{Spec}(B)) \cong \operatorname{Hom}_{\mathrm{CAlg}(\mathrm{Sp})}(B, \mathrm{~S}),(7.7)
$$

Proof. The demonstration is very immediate using the mentioned properties inside the hypothesis of the Theorem. However is necessary establish some fine details on the acting of the functors F, G, that appear in the Koszul duality application and the relative details on the inverse limits to obtain Spf, ${ }^{4}$ in the context of "CRings", $\mathrm{CAlg}(\mathrm{Sp})$.

\footnotetext{
${ }^{4}$ If $A \cong \lim _{\alpha} A_{\alpha}$, is a pro-object of $\operatorname{Alg}_{\text {sm }}^{(n)}$, we let the functor

$\operatorname{Spf}(A): \operatorname{Alg}_{\mathrm{sm}}^{(n)} \rightarrow \mathrm{S}$,

as the functor given by the formula
} 
Indeed, if $A \cong \lim _{\leftarrow} A_{\alpha}$, is a pro-object of $\operatorname{Alg}_{\mathrm{sm}}^{(n)}$, then

$$
\operatorname{Spf} A \cong \underset{\alpha}{\lim } \operatorname{Spec} A
$$

then $\operatorname{SpfA} \in$ Moduli $_{n}$.

For other side, considering $A$, a $E_{n}$-small algebra over $k$, and $\operatorname{Spec} A \in \operatorname{Moduli}_{n}$, denoting the representation functor $\Phi^{-1}: \operatorname{Alg}_{\mathrm{sm}}^{(n)} \rightarrow \mathrm{S}$, given by the formula

$$
\begin{aligned}
& (\operatorname{Spec} A)(B)=\operatorname{Hom}_{\operatorname{Alg}_{\mathrm{sm}}^{(n)}}(A, B)=\Psi(\mathrm{D} A)(B) \\
& =\mathrm{G} \Psi(B)=\mathrm{G \Psi}_{\mathrm{R}} \in \operatorname{Spec} B \in \operatorname{Fun}\left(\operatorname{Alg}_{\mathrm{sm}}^{(n)}, \mathrm{S}\right),
\end{aligned}
$$

To the functor $\mathrm{F}$, the existence of an arbitrary element $\operatorname{Spec} B$, that fall inside of $\operatorname{Alg}_{\text {aug }}^{(n)}$ needs the additional arguments as Moduli $_{n}^{\circ}=$ Moduli $_{n}$, which was mentioned before. Then the compute of the functor $\Psi$, can be realized easily .

Then by (7. 1) all equivalences are satisfied in the context of the moduli schemes and "CRings". Due to that the functor $\Phi:$ Moduli $_{n} \rightarrow \mathrm{Alg}_{\text {aug }}^{(n)}$, is faithful then is followed the all scheme (7. 7).

\section{Integral Transforms Applications}

As mentioned in the before section 7, the left and right functors F, G, appear by the integral transforms that are involved in the $k$-modules level. If we consider that these $k$ - modules are $D_{G / H}-$ modules then the equivalences given by the Penrose transform [16]

$$
H^{0}\left(X, \mathrm{~L}_{\lambda}\right) \cong \operatorname{ker}\left(\widetilde{U}, Q_{B R S T}\right)
$$

are translated in the equivalences $[16,17]$ :

$$
\mathrm{M}\left(D_{G / H}-\text { modules } G \text {-equivariants }\right) \underset{G \Psi_{\mathrm{R}}}{\stackrel{\Phi_{\mathrm{R}} \mathrm{F}}{S}} \mathrm{M}_{G}(\mathrm{~g}, H),
$$

which are translated in the isomorphism the Hecke categories [16]:

$$
\mathrm{H}_{G^{\wedge}} \Delta \mathrm{M}(\widetilde{\mathrm{g}}, \mathrm{Y})
$$

where The Lie algebra $\widetilde{g}$, is the loop extension of the loop algebra $\mathrm{g}(t)$.

Considering the role of $S$, in the theory of $\infty$ - categories as the analogous to that of the ordinary category of sets in classical category theory, and the Yoneda embedding defined by (4. 1) and (4.2) for any $\infty$ - category $C$, then in particular

$$
B \mapsto \operatorname{Hom}_{\mathrm{Pro}\left(\mathrm{Alg} \mathrm{g}_{\mathrm{m}}^{(n)}\right)}(A, B) \cong \underset{\alpha}{\operatorname{Lim}} \operatorname{Hom}_{\mathrm{Alg}_{\mathrm{m}}^{(n)}}\left(A_{\alpha}, B\right)
$$

to a graded algebra $H^{\bullet}\left(B_{\text {Bun }}, \mathrm{D}^{s}\right)$, obtained from a Yoneda embedding, and generated by one copy of $\mathrm{H}^{\vee}$, over $\left.H^{0} \cong \mathrm{Q}^{0} \mathrm{pp}_{{ }^{L_{G}}}\right]$, is had that on a disk that [18]:

Theorem (E. Frenkel, C. Teleman) 8. 1. The Yoneda algebra $\operatorname{Ext}_{\mathrm{D}^{s}\left(\mathrm{Bun}_{G}\right)}\left(\mathrm{D}^{s}, \mathrm{D}{ }^{s}\right)$, is abstractly $A_{\infty}$ - isomorphic to (the strictly skew-commutative one) $\mathrm{Ext}_{\mathrm{Loc}_{G}^{L}{ }_{G}}\left(\mathrm{O}_{\mathrm{Op}_{G}^{L}}, \mathrm{O}_{\mathrm{Op}_{G}^{L} G}\right)$.

Considering a full subcategory of sheaves in $\mathrm{C}=\operatorname{Coh}\left(\operatorname{Loc}_{L_{G}}\right)$, then we have:

$$
H^{\bullet}\left(\mathrm{g}[[z]], \mathrm{g} ; \mathrm{V}_{\text {crit }}\right) \cong \Omega^{\bullet}\left[\mathrm{Op}_{{ }_{G}}(D)\right]
$$

Then considering an $A_{\infty}$ - enhancement of (8.4), that is to say, an $\infty$-algebra in $\mathrm{Alg}_{\text {aug }}^{(n)}$, then we can give the isomorphism

$$
H^{0}\left(\mathrm{~g}[[z]] ; \mathrm{V}_{\text {crit }}\right) \cong \operatorname{ker}\left(\operatorname{Bun}_{G}^{\circ}, \partial\right),
$$

which is the Penrose transform to a functor Fun(D top,$S)$. Here $\operatorname{Bun}_{G}{ }_{G}=\mathrm{X}$, where $\mathrm{X}$, is the flag variety as the "quantum" version of the construction of an algebra symT.

\section{Acknowledgements}

I am very grateful to the research group for the invitation to participate in the seminar that organized in Chalco, Mexico the Lead Guest Editor of the special issue in integral geometry methods on derived categories in the geometrical Langlands program.

\section{References}

[1] A. Grothendieck, On the De Rham Cohomology of algebraic varieties, Publ. Math.I.H.E.S. 29 (1966) 95-103.

[2] M. Kontsevich, Y. Soibelman, Deformations of algebras over operads and the Deligne conjecture, Conference Moshe Flato 1999, Vol 1 (Dijon), 255-307, Math. Phys. Stud., 21, Kluwer Acad. Publ., Dordrecht, 2000.

[3] J. Milnor, "On spaces having the homotopy type of a CW-complex" Trans. Amer. Math. Soc. 90 (1959), 272-280.

[4] C. Teleman, The quantization conjecture revised, Ann. Of Math. (2) $152(2000), 1-43$.

[5] P. G. Goerss, "Topological modular forms (aftern Hopkins, Miller, and Lurie)," Available at arXiv:0910.5130v1

[6] A. Efimov, V. Lunts, D. Orlov, Deformations theoryof objects in homotopy and derived categories I: General Theory, Adv. Math. 222 (2009), no. 2, 359-401.

[7] A. Efimov, V. Lunts, D. Orlov, Deformations theoryof objects in homotopy and derived categories II: Pro-representability of the deformation functor, Available at arXiv:math/0702839v3.

[8] A. Efimov, V. Lunts, D. Orlov, Deformations theoryof objects in homotopy and derived categories III: Abelian categories, Available at arXiv:math/0702840v3. 
[9] V. Hinich, DG coalgebras as formal stacks, J. Pure Appl. Algebra, 162 (2001), 209-250.

[10] V. Hinich, DG Deformations of homotopy algebras, Communications in Algebra, 32 (2004), 473-494.

[11] B.Toën,The homotopy theory of dg-categories and derived Morita theory, Invent. Math. 167 (2007), no. 3, 615-667.

[12] B.Toën, M. Vaquié, Moduli of objects in dg-categories, Ann. Sci. cole Norm. Sup. (4) 40 (2007), no. 3, 387-444.

[13] B.Toën, G. Vezzosi, From HAG to derived moduli stacks. Axiomatic, enriched and motivic homotopy theory, 173-216. NATO Sci. Ser II, Math. Phys. Chem., 131, Kluwer Acad. Publ. Dordrecht, 2004.

[14] D. Ben-zvi and D. Nadler, The character theory of complex group, 5 (2011) arXiv:0904.1247v2[math.RT],

[15] B. Fresse, Koszul duality of $E_{n}$-operads, Available as arXiv:0904.3123v6

[16] F. Bulnes, Penrose Transform on Induced $D_{G / H}$-Modules and Their Moduli Stacks in the Field Theory, Advances in Pure Mathematics 3 (2) (2013) 246-253. doi: 10.4236/apm.2013.32035.

[17] F. Bulnes, Cohomology of Moduli Spaces in Differential Operators Classification to the Field Theory (II), in: Proceedings of Function Spaces, Differential Operators and Non-linear Analysis., 2011, Tabarz Thur, Germany, Vol. 1 (12) pp001-022.

[18] E. Frenkel, C. Teleman, Geometric Langlands Correspondence Near Opers, Available at arXiv:1306.0876v1. 